Trauma Berufskrankh 2009 · 11 [Suppl 2]: 154-159

DOI 10.1007/s10039-009-1504-3

Online publiziert: 18. Juni 2009

(c) Springer Medizin Verlag 2009

\author{
E. Bobrich $\cdot$ C. Haupt $\cdot$ R. Grass $\cdot$ H. Zwipp \\ Klinik und Poliklinik für Unfall- und Wiederherstellungschirurgie, \\ Universitätsklinikum Carl Gustav Carus, TU Dresden
}

\section{Tibiakopffraktur und Luxationsfraktur}

\section{Ätiologie und Epidemiologie}

In Zusammenschau aller Tibiafrakturen treten Brüche des proximalen Drittels mit einer Inzidenz von etwa 10\% auf, sie stellen damit $<1 \%$ aller knöchernen Verletzungen dar [4]. Dabei ist ein Häufigkeitsgipfel im 50.-70. Lebensjahr auffällig. Niedrigenergietraumen durch Freizeit- und häusliche Unfälle treten gehäuft bei Frauen höheren Alters auf, während Hochenergietraumen nach Verkehrs- und Arbeitsunfällen eher bei jungen Männern beobachtet werden.

Tibiakopffrakturen kommen in einer Vielzahl von Formen vor und entstehen durch direkte Gewalteinwirkung [21], v. a. bei Rasanztraumen, durch indirekte Gewalteinwirkung (Varus- oder Valgusstress), Rotation sowie in der Regel aus der Kombination einer axialen Kompression mit einer Valgisierung oder Varisierung [7].

Entsprechend der Intensität der einwirkenden Kraft, die bis zu 5882 N (6oo kp) erreichen kann [6], unterscheiden sich Art der knöchernen Läsion, Dislokation der Fragmente und Begleitverletzungen.

An erster Stelle der Unfallursachen werden in der Literatur Verkehrsunfälle (Motorrad, PKW, Fußgänger) genannt [18], gefolgt von Stürzen aus größerer Höhe (häusliche und Arbeitsunfälle) sowie Sport- und Freizeitunfälle (Ski).

\section{Begleitverletzungen}

Tibiakopffrakturen stellen in der Regel Gelenkfrakturen dar. Aufgrund der ana- tomischen Verhältnisse lassen sich häufig Begleitverletzungen im Sinne von Ligament- und Meniskusverletzungen sowie Fibulaköpfchenfrakturen finden, die primär aufgrund der Schwellung und unzureichender schmerzbedingter klinischer Beurteilung oft nicht erkannt werden [5].

Wichtige und häufige Komplikationen der Tibiakopffraktur, welche mit einer Inzidenz von $20 \%$ beschrieben wurden [10], sind Läsionen der A. und V. poplitea und des N. peronaeus communis. Dabei kommt es bei einer akuten Unterbrechung des Blutstromes in der A. poplitea durch Abriss, Verschluss oder Verlegung durch einen Thrombus in $49 \%$ der Fälle zu einer kompletten Ischämie mit drohender Amputation des Unterschenkels [8]. Die Häufigkeit der Verletzung des N. peronaeus im Bereich des Fibulaköpfchens wurde in der Literatur mit 2\% [19] und 6,8\% [23] angegeben.

Aus der anatomischen Struktur des Kniegelenkes erklärt sich das gehäufte Auftreten von Kniebandverletzungen mit einer in der Literatur beschriebenen, weit gefächerten Inzidenz von 2,5-54,8\% [3].

Bei Luxationsfrakturen und Distorsionstraumen des Kniegelenkes finden sich zusätzlich gehäuft Kapsel-Band-Verletzungen [9]. In einer 1990 veröffentlichten Studie wiesen von 315 Patienten mit Tibiakopffrakturen 21,9\% eine Kniebandverletzung auf. Dabei wurden bei $49,3 \%$ isolierte Schädigungen des medialen und bei $31,9 \%$ des lateralen Seitenbandes sowie bei 5,8\% eine Verletzung des vorderen Kreuzbandes gefunden [2].
Meniskusläsionen stellen ebenfalls eine relativ häufige Begleitverletzung bei Tibiakopffrakturen dar. Nicolet [12] wies auf eine 90\%ige Inzidenz der lateralen Meniskusverletzungen aufgrund der anatomische Form sowie der häufiger vorkommenden lateralen Kondylenbrüche hin, während mediale Meniskusläsionen mit einer Häufigkeit von $20 \%$ vorkommen [12]. Die unterschiedlichen Angaben der Literatur über die Inzidenz der Meniskusverletzungen bei Tibiakopffrakturen wurden in der Studie von Dustmann et al. [4], die eine Variation der Inzidenz von 12,5$25 \%$ berichteten, noch einmal verdeutlicht.

Die Häufigkeit von offenen Frakturen, Weichteilschäden und Kompartimentsyndromen bei Tibiakopffrakturen wird in der Literatur mit einer Prävalenz von o,o$8,0 \%$ angegeben [18][20].

Die wichtigste Komplikation nach der Behandlung offener Frakturen stellt die Infektion dar. Zudem kann es zum Ansteigen der Inzidenz einer verzögerten Knochenheilung bzw. Entwicklung einer Pseudarthrose kommen, die in Kombination mit einem infektiösen Geschehen im schlimmsten Fall eine Amputation zur Folge haben kann [14].

\section{Frakturklassifikationen}

In der Literatur findet sich kein einheitliches Klassifikationssystem der Tibiakopffrakturen, entsprechend schwierig ist der Vergleich von Verletzungsmustern und therapeutischen Empfehlungen. 


\section{Tibiakopffrakturen}

\section{AO-Klassifikation}

Die von der Arbeitsgemeinschaft für Osteosynthese $(\mathrm{AO})$ vorgeschlagene und im deutschsprachigen Raum sehr verbreitete Klassifikation, die initial auf einer Einteilung von Müller u. Allgöwer [11] beruhte, orientiert sich an der radiologischen Frakturmorphologie. Dabei werden extraartikuläre knöcherne Ligamentausrisse und metaphysäre A-Frakturen von unikondylären, intraartikulären Spalt-, Impressions- sowie unikondylären Kombinationsfrakturen der Gruppe B unterschieden. In der Gruppe $C$ werden bikondyläre sowie Trümmerfrakturen zusammengefasst. Die zusätzliche Einteilung $(1,2,3)$ beschreibt den Grad der Fragmentierung (• Abb. 1, 2).

Kennzeichnend für die AO-Klassifikation ist ihr Aufbau nach morphologischen Gesichtspunkten. Bestimmte typische Frakturformen am Tibiakopf sowie das Erkennen von gleichzeitig vorliegenden ligamentären Läsionen sowie Weichteilschäden werden nicht berücksichtigt.

\section{Klassifikation nach Tscherne u. Lobenhoffer}

Hier werden die Tibiakopffrakturen unter Berücksichtigung von Entstehungsmechanismus, Instabilität sowie zu erwartendem Weichteilschaden differenziert in [22]:

- Plateaufrakturen,

- Luxationsfrakturen und

- Trümmerfrakturen.

Plateaufrakturen entstehen dabei durch ein axiales Stauchungstrauma (Sturz oder leichter Sportunfall), was zu einer Spaltung oder Impression eines oder beider Tibiaplateaus mit oder ohne Depression führt. Aufgrund des Unfallmechanismus ist die Häufigkeit von begleitenden Kapsel-Band-Verletzungen gering.

Für die Plateaufrakturen wurde von Tscherne u. Lobenhoffer [22] ein eigenständiges Klassifikationssystem entwickelt (ठ Abb. 3). Dass sich dieses bei genauer Betrachtung im Klassifikationssystem der $\mathrm{AO}$ widerspiegelt ( $\mathrm{P}_{1}$ : unikondyläre Spaltfraktur, entspricht AO- $\mathrm{B}_{1} ; \mathrm{P}_{2}$ : unikondyläre Impressionsfraktur, entspricht $\mathrm{AO}-\mathrm{B}_{2}$; $\mathrm{P}_{3}$ : Kombinationsfraktur, entspricht $\mathrm{AO}_{-} \mathrm{B}_{3}$; $\mathrm{P}_{4}$ : bikondyläre Frak-

Trauma Berufskrankh 2009 · 11 [Suppl 2]: 154-159 DOI 10.1007/s10039-009-1504-3

(c) Springer Medizin Verlag 2009

\section{E. Bobrich · C. Haupt $\cdot$ R. Grass $\cdot$ H. Zwipp Tibiakopffraktur und Luxationsfraktur. Klassifikation und Begleitverletzungen}

\section{Zusammenfassung}

Aufgrund der speziellen Anatomie des Kniegelenkes, der Nähe zu Nerven und Gefäßen, dem Bandapparat und den Muskeln ist eine Vielzahl an Begleitverletzungen und Komplikationen möglich. Luxations- und Trümmerfrakturen, die beim jugendlichen Patienten im Rahmen von Rasanztraumen auftreten, sind oft mit Bandläsionen und neurovaskulären Verletzungen vergesellschaftet sowie hochgradig instabil. Neben Schäden $\operatorname{der} A$. und V. poplitea sowie des N. peronaeus communis, die in der Literatur mit bis zu 20\% angegeben werden, treten Bandläsionen mit einer Häufigkeit von 20-25\% auf. Die Inzidenz von Meniskusverletzungen bei Tibiakopffrakturen beträgt $12-25 \%$. Nicht zu vernachlässigen sind posttraumatische Kompartimentsyndrome sowie Weichteilschäden, die das funktionelle Ergebnis deutlich beeinflussen. Zur morphologischen Einteilung der Frakturtypen wurde eine Vielzahl von Klassifikationssystemen publiziert. Unter Berücksichtigung der ligamentären und neurovaskulären Begleitverletzungen erscheint eine Einteilung in Plateau- und Luxationsfrakturen sinnvoll. Die AO-Klassifikation umfasst insbesondere die Plateaufrakturen, die Luxationsfrakturen werden im Klassifikationssystem nach Moore dargestellt, Überschneidungen der beiden Systeme sind vorhanden.

\section{Schlüsselwörter}

Tibiakopffraktur · Luxationsfraktur · Begleitverletzung · Komplikation · Frakturklassifikation

\section{Tibial plateau fractures and luxation fractures. Fracture classification and associated lesions}

\section{Abstract}

Due to the complex anatomy of the knee joint, its proximity to nerves, vessels, ligament structures and muscles, there is a wide range of possible complications and associated lesions. Luxation fractures and comminuted fractures, which occur in young patients in the context of high-speed traumas, are often associated with ligament lesions and injuries of neurovascular structures. They are also extremely unstable. In addition to vascular and peroneal nerve injuries, which are described in the literature as occurring with a frequency of up to $20 \%$, ligament lesions occur with a frequency of $20-25 \%$. The incidence of meniscus injuries is given as $12-25 \%$. The posttraumatic appearance of compartment syndrome, as well as soft tissue damage, is not to be neglected, having a significant influence on the functional outcome. A considerable number of classification systems for the morphological division of the fracture types are given in the literature. Given the ligament and neurovascular nature of accompanying injuries, a division into plateau and luxation fractures seems sensible. The AO classification includes in particular plateau fractures, while luxation fractures are shown in the classification system according to Moore. Overlaps between the classification systems exist.

\section{Keywords}

Tibial plateau fractures - Luxation fractures . Associated lesions · Complications · Fracture classification 


\section{Knieverletzungen}

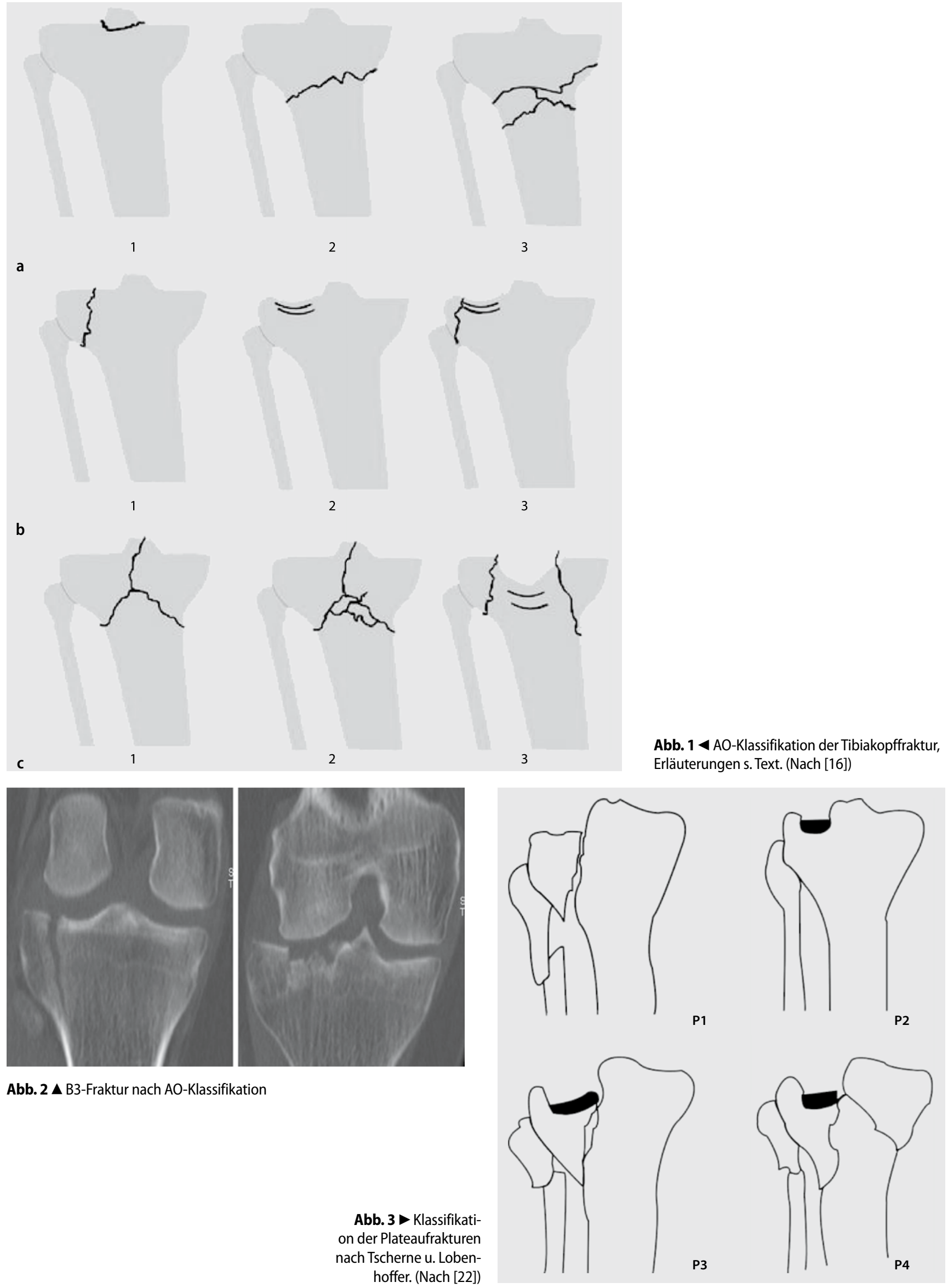




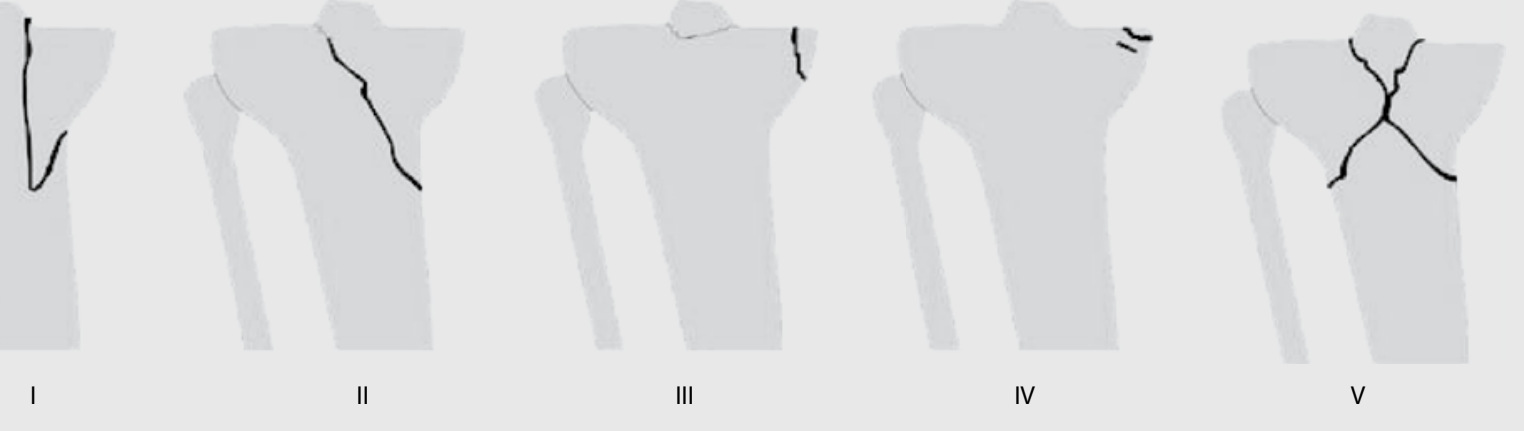

Abb. 4 \ Klassifikation der Luxationsfrakturen nach Moore. (Nach [15])

turen, entspricht $\left.\mathrm{AO}-\mathrm{C}_{3}\right)$ (Vgl. $\bullet$ Abb. 1 und 3), mag neben dem unzureichenden Erkennen von Begleitverletzungen ein Grund dafür sein, warum die Klassifikation nach Tscherne $\mathrm{u}$. Lobenhoffer [22] im klinischen Alltag nur selten Gebrauch findet.

\section{Luxationsfrakturen}

Sie resultieren aus Rotations- und Verrenkungstraumen mit erheblichen Rotations- und Scherkräften, sodass Verletzungen dieser Art beispielsweise nach Hochrasanztraumen oder direktem Anprall häufig mit femorotibialen Luxationen und Subluxationen vergesellschaftet sind, aus denen femorotibiale Instabilitäten resultieren [5]. Aufgrund der Häufigkeit von assoziierten Begleitverletzungen, welche bei Patienten mit undislozierten oder bereits reponierten Frakturen initial schnell übersehen werden können, ist das Verständnis des Gesamtausmaßes dieser Frakturen zur Einleitung eines adäquaten Behandlungsregimes dringend erforderlich.

\section{Moore-Klassifikation}

Moore [10] stellte anhand röntgenologischer Stressaufnahmen von 1000 Tibiakopffrakturen ein Klassifikationssystem für Luxationsfrakturen auf, welches die Gelenkinstabilität vergesellschaftet mit einer hohen Inzidenz an ligamentären und neurovaskulären Verletzungen darstellt (• Abb. 4).

Über seine Klassifikationsmethodik sollte eine bessere präoperative Einschätzung der erwarteten ligamentären, neurovaskulären und meniskusassoziierten Schäden am Kniegelenk gelingen. Moore [10] teilte die Luxationsfrakturen in 5 Typen ein, wobei Typ 2 und 4 nochmals in

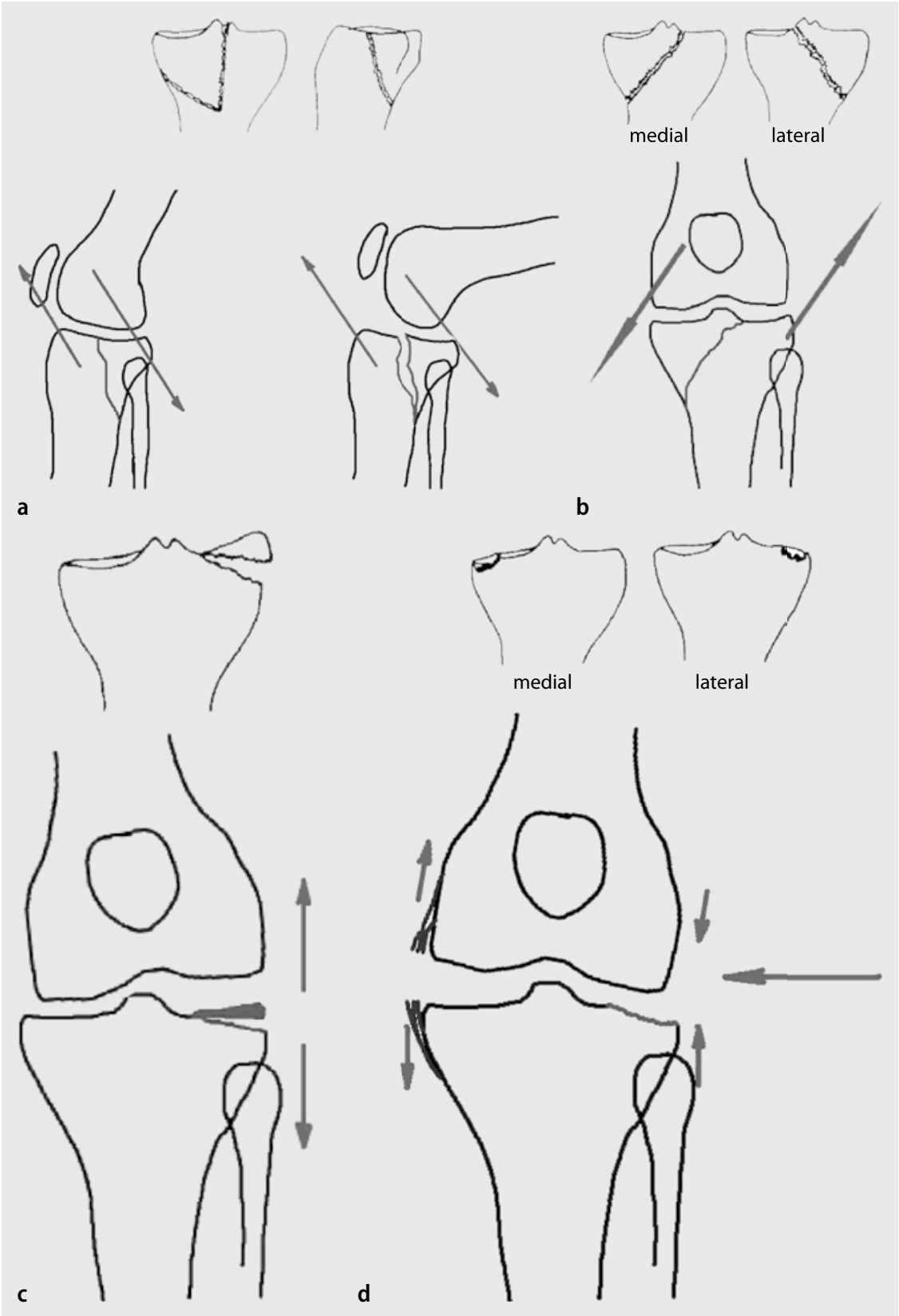

Abb. 5 \ Unfallmechanismen, Moore-Klassifikation, a Typ-I-Fraktur, b Typ-II-Fraktur, c Typ-III-Fraktur, d Typ-IV-Fraktur 

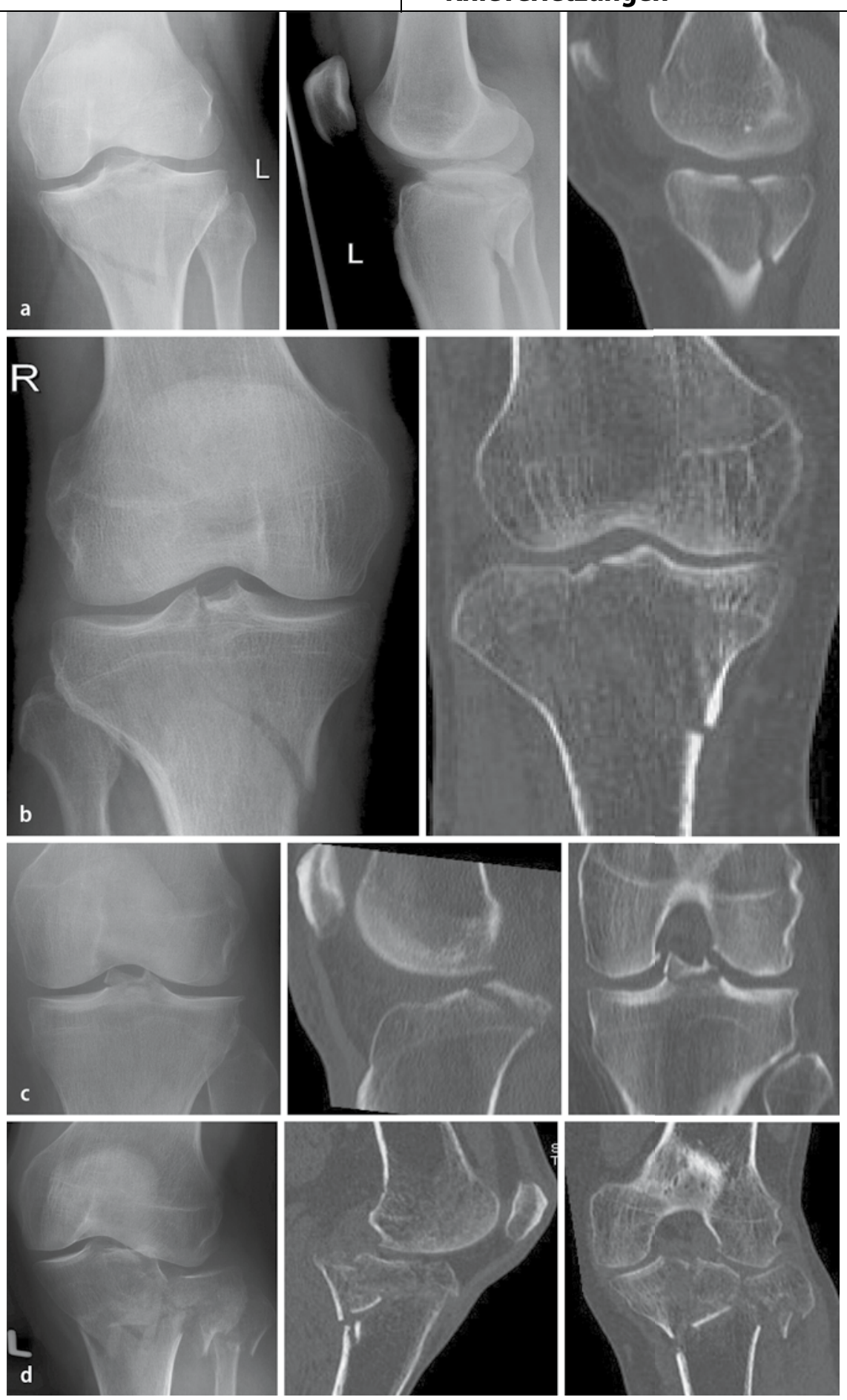

Abb. 6 \ Röntgenbilder, Moore-Klassifikation, a Typ-I-Fraktur, b Typ-II-Fraktur, c Typ-III-Fraktur, d TypV-Fraktur

eine mediale und eine laterale Form unterschieden werden.

Typ I nach Moore. Dieser Frakturtyp, der dorsale mediale Kondylenspaltbruch ("medial split fracture"), umfasst das ganze mediale Kompartiment in der a.-p. und mindestens die Hälfte der Gelenkfläche in der seitlichen Projektion. Bei Knie-
Typ II nach Moore. Er ist charakterisiert als "entire condyle fracture" (kompletter Kondylenbruch), wobei die Frakturlinie bis in das kontralaterale Tibiaplateau unter vollständigem/teilweisem Einschluss der Eminentia intercondylaris ausläuft (- Abb.5b, 0 Abb. 6b). Ist diese nicht separiert, muss eine ligamentäre Ruptur eines oder beider Kreuzbänder vorliegen.

Die Rate an neurovaskulären Begleitläsionen ist bei diesem Frakturtyp mit nahezu 20\% substanziell [10].

Typ III nach Moore. Hierunter ("rim avulsion fracture") werden knöcherne Randausrisse im Plateaubereich zusammengefasst, die häufiger im lateralen Kompartiment gesehen werden (- Abb. 5c, - Abb. 6c). Ein Beispiel ist die von Segond [17] beschriebene ossäre Avulsion der Gelenkkapsel im mittleren Drittel des lateralen Tibiaplateaus dorsal des Tubercule de Gerdy. Andere Beispiele sind Avulsionsfrakturen des Fibulaköpfchens, Rupturen des lateralen Seiten- und/oder hinteren Kreuzbandes sowie basisnahe Meniskusabrisse.

Die Rate der neurovaskulären Begleitverletzungen beträgt 30\% [10].

Typ IV nach Moore. Die hierunter verstandene "rim impression" ist als Zeichen einer stattgehabten Luxation zu werten und tritt medial und lateral auf. Im Rahmen des Luxationsmechanismus kommt es zu einer Impaktion des unter dem Meniskus gelegenen Kantenfragmentes mit gleichzeitiger Bandverletzung der Gegenseite (• Abb. 5d).

Die Inzidenz der neurovaskulären Läsionen ist im Vergleich zu den Verletzungen des Bandapparates gering.

Typ V nach Moore. Charakteristisch für diesen Frakturtyp, die "four-part-fracture" (- Abb. 6d), ist die Trümmerfraktur mit separatem Ausriss der Eminentia intercondylaris (im Vergleich zur bikondylären Plateaufraktur).

Die Separation der Eminentia vom Schaft sowie von beiden Kondylen führt zu einer ausgeprägten Instabilität sowie einer hohen Inzidenz an neurovaskulären Begleitverletzungen (50\% nach Moore [10]. 


\section{Resümee}

Entsprechend der beschriebenen Frakturformen der Luxationsfrakturen wird deutlich, dass anhand der Kenntnis des Unfallmechanismus in Zusammenschau mit der klinischen sowie röntgenologischen Frakturmorphologie schneller Kenntnisse über die Begleitverletzungen gewonnen und ein geeignetes Therapiekonzept zügiger entworfen werden können.

\section{Fazit}

Tibiakopffrakturen stellen im klinischen Alltag häufige Verletzungen dar. Aufgrund der anatomischen Verhältnisse am Kniegelenk sind Begleitverletzungen häufig.

Das primäre radiologische Erscheinungsbild ist nicht unbedingt aussagekräftig bezüglich des tatsächlichen Frakturausmaßes sowie korrespondierender Begleitverletzungen. Zur standardisierten Wahl adäquater Therapieoptionen wurden verschiedene Klassifikationssysteme entwickelt. Dabei haben sich die Klassifikationen der Tibiakopffrakturen zum einen nach radiologisch-morphologischen Kriterien im Hinblick auf die Plateaufrakturen (AO-Klassifikation), zum anderen nach funktionellen Gesichtspunkten bezogen auf die Luxationsfrakturen (Moore-Klassifikation) nebeneinander im klinisch-chirurgischen Alltag durchgesetzt.

\section{Korrespondenzadresse}

\section{Dr. E. Bobrich}

Klinik und Poliklinik für Unfallund Wiederherstellungschirurgie, Universitätsklinikum Carl Gustav Carus, TU Dresden,

Fetscherstraße 74, 01307 Dresden

eva.bobrich@uniklinikum-dresden.de

Interessenkonflikt. Keine Angaben

\section{Literatur}

1. Beharry R, McKnee MD (2001) Plateau fractures. J Orthop Trauma 15:312-320

2. Delamarter RB, Hohl M, Hopp E Jr (1990) Ligament injuries associated with tibial plateau fractures. Clin Orthop 250:226-233

3. Dickob M, Mommsen U (1994) Tibiakopffrakturen und Kniebandverletzungen. Unfallchirurgie 20:8893
4. Dustmann HO, Schulitz KP, Heinzmann J (2007) Konservative oder operative Behandlung von Tibiakopffrakturen. Orthop Unfallchir up2date 2:377396

5. Kotter A, Rueter A (1997) Tibiakopffrakturen. Unfallchirurg 100:742-749

6. Krettek C, Schandelmaier $P$, Lobenhoffer $P$, Tscherne H (1996) Komplextrauma des Kniegelenkes. Diagnostik - Management - Therapieprinzipien. Unfallchirurg 99:616-628

7. Kuner E, Schlosser V (Hrsg) (1995) Traumatologie, 5. Aufl. Thieme, Stuttgart

8. Lobenhoffer P, Krettek C (1997) Das komplexe Kniegelenktrauma. Orthopade 26:1037-1045

9. Lobenhoffer P, Oestern HJ (1997) Möglichkeiten minimal-chirurgischer Maßnahmen am Kniegelenk. Chirurg 68:1093-1105

10. Moore TM (1981) Fracture-dislocation of the knee Clin Orthop 156:128-134

11. Müller ME, Allgöwer M (1977) Manual der Osteosynthese, 2. Aufl. Springer, Heidelberg

12. Nicolet A (1965) Die Meniskusverletzung der Tibiakopffrakturen. Langenbecks Arch Chir 313:544549

13. NN (1973) Schienbeinkopfbrüchen. Z Orthop Ihre Grenzgeb 111:160-168

14. Ostermann PAW (2001) Weichteilschaden - Strategie zur Infektvermeidung. Trauma Berufskrankh 3:174-176

15. Petersen W, ZantopT (2006) Tibiakopffraktur. Unfallchirurg 109:219-232

16. Raschke M, Zantop T (2007) Tibiakopffraktur. Chirurg 78:1157-1171

17. Segond P (1879) Recherches cliniques et experimentales sur les epanchements sanguins du genou par entorse. Paris Progres Med 297-421

18. Stevens DG, Beharry R, McKee MD et al (2001) The long-term functional outcome of operatively treated tibial plateau fractures. J Orthop Trauma 15(5):312-320

19. Thiele K (1968) Schienbeinkopfbrüche: Bruchformen, Behandlung, Spätergebnisse bei 486 Fällen. Hefte Unfallheilkd 95:1-126

20. Tralles D, Wojczik H (1990) Auswertung einer DDRSammelstudie. Zentralbl Chir 115:635-643

21. Trentz O, Ertel W (1995) Unfallchirurg, 10. Aufl. Urban \& Fischer, München

22. Tscherne H, Lobenhoffer P (1993) Tibial plateau fractures. Management and expected results. Clin Orthop 292:87

23. Welte G (1984) Früh- und Spätergebnisse operativ versorgter Tibiakopffrakturen. Med. Dissertation, Universität Tübingen 